\title{
Study of the Chromosomal Abnormalities and Associated Complex Karyotypes in Hematological Cancer in the Population of West Bengal: A Prospective Observational Study
}

Puspal De, Madhumitha J. Mukhopadhyay

'Department of Genetics, Institute of Genetic Engineering, Kolkata, West Bengal, India

Ind J Med Paediatr Oncol 2021;42:261-267.
Address for correspondence Mr. Puspal De, MSc, MPhil, Department of Genetics, Institute of Genetic Engineering, 30 Thakurhat Road, Kolkata, 700128, West Bengal, India (e-mail: puspal.dey@gmail.com).

\begin{abstract}
Keywords

- chromosomal instability

- hematological cancer

- complex karyotype

- leukocyte culture

Introduction Chromosomal instability is an important feature of hematological cancer. The pathogenesis is complex and it involves genetic and epigenetic factors. As a genetic factor, chromosomal instability may play a key role in leukemogenesis. Accumulation of genetic alteration is mainly responsible for numerical and structural chromosomal rearrangement or clonal evaluation. But disease progression is often driven by chromosomal translocation, hyper- or hypodiploidy with structural abnormalities, and complex karyotypes.

Objective This research aimed to study the different types of chromosomal abnormalities in clinically suspected hematological cancer patients.

Materials and Methods Cytogenetic analysis was performed based on phytohemaglutinin stimulated peripheral blood lymphocyte cultures and bone marrow culture, without mitogen, of the respective patients of West Bengal from March 2016 to February 2018. All clinically suspected hematological cancer patients referred for karyotyping to the institutional genetics department have been included without any biasness of sex and age. Karyotypes were described according to the International System for Cytogenetic Nomenclature (ISCN 2005).

Results In the present study, 56 clinically suspected hematological cancer cases were observed and 41 cases of chromosomal rearrangement were found which clearly show chromosomal instability as the main driving force for hematological cancer transformation. Presence of variant Philadelphia chromosomes with classical translocation, mosaic complex karyotypes, variable numerical, and structural chromosomal abnormality, along with severe-to-moderate hypo- and hyperdiploidy, and presence of marker chromosomes were the main findings of this study.

Conclusion The result shows that the detection of chromosomal instability was important for preliminary diagnosis, treatment, prognosis, and further management. So the present study provided additional information about chromosomal instability in hematological cancer at Kolkata and adjoining regions.
\end{abstract}

DOI https://doi.org/ 10.1055/s-0041-1733827 ISSN 0971-5851
C 2021. Indian Society of Medical and Paediatric Oncology. This is an open access article published by Thieme under the terms of the Creative Commons Attribution-NonDerivative-NonCommercial-License, permitting copying and reproduction so long as the original work is given appropriate credit. Contents may not be used for commercial purposes, or adapted, remixed, transformed or built upon. (https://creativecommons.org/licenses/by-nc-nd/4.0/). Thieme Medical and Scientific Publishers Private Ltd. A-12, Second Floor, Sector -2, NOIDA -201301, India 


\section{Introduction}

Cancer progression involves a cascade of phenomena and each step shows varying histopathological stages. ${ }^{1}$ Accumulation of genetic alterations which ultimately cause subsequent changes in gene expression are the main driving forces of cancer progression. ${ }^{2}$ To understand the biology of the process, identification of the altered gene and determination of exact gene expression pathway are important steps. It also helps in early diagnosis to explore new strategies for treatment and further management for the patient's recovery. ${ }^{3}$ The cytogenetic approach for detection of chromosomal abnormality and identification of the disrupted gene in cancer development has become the most effective approach.

The accumulation of genetic alterations converts normal cells into invasive cancerous cells and finally transforms metastatic disease through hyperplastic and dysplastic stages. In premalignant stages of tumor, genetic instability is more common which promotes cancer development. Finally, the complex patterns of genetic alteration create an unstable genomic condition and in the later stage of disease, transform tumor cells from premalignant stage to malignancy. So, all cancer cells have some alteration of gene expression or functions. ${ }^{4,5}$ The genetic alteration includes numerical chromosomal abnormality such as aneuploidy and severe-to-moderate hypo- and hyperdiploidy and structural chromosomal abnormalities like chromosomal translocations, inversions, or loss of the whole chromosome. ${ }^{6}$

Nonrandom chromosomal abnormalities have important biological, diagnostic, and prognostic significance in hematological cancers. Numerical chromosomal abnormality and structural chromosomal abnormality are the common forms of chromosomal abnormalities found in chromosomal disorders. Generally, either any one type of chromosomal abnormality is found in an individual, but in case of patients suffering from hematological disorders, the karyotypes may contain both types of changes. ${ }^{6,7}$

To assess the percentage of chromosomal rearrangements or chromosomal alterations in hematological cancers, the present study was conducted with association of a tertiary care hospital, in Kolkata, over a period of 2 years. During this period, a total of 56 suspected hematological cancer cases were cytogenetically evaluated. After clinical

Table 1 The clinically defined types of the hematological cancers and the sex of the patients

\begin{tabular}{|l|l|l|}
\hline \multirow{2}{*}{$\begin{array}{l}\text { Types of clinically diagnosed } \\
\text { hematological cancer }\end{array}$} & \multicolumn{2}{|c|}{$\begin{array}{c}\text { Sex of the suspected } \\
\text { individual }\end{array}$} \\
\cline { 2 - 3 } & Male & Female \\
\hline $\begin{array}{l}\text { CML = chronic myelogenous } \\
\text { leukemia }\end{array}$ & 25 & 17 \\
\hline CLL= chronic lymphocytic leukemia & 00 & 00 \\
\hline AML= acute myelogenous leukemia & 04 & 03 \\
\hline ALL= acute lymphoblastic leukemia & 03 & 04 \\
\hline Undiagnosed & 02 & 00 \\
\hline
\end{tabular}

aSuspected hematological cancer but specific type was not determined by the clinician. investigation by expert clinicians, the peripheral blood and bone marrow samples were collected from the suspected patients and then processed in our cytogenetic laboratory for chromosomal study. The chromosome analysis revealed normal chromosome constituents along with classical Philadelphia chromosomes, variant Philadelphia chromosomes, complex karyotypes, hyperdiploidy, hypodiploidy, and metaphases with maker chromosomes. Thus, cytogenetic evaluation remains the preliminary technique to detect the spectrum of chromosomal rearrangement in hematological cancers.

\section{Materials and Methods}

For peripheral blood culture, RPMI-1640 cell culture liquid media with L-glutamine and sodium bicarbonate was taken from Sigma-Aldrich (R8758-500ML), phytohemaglutinin or PHA is collected from Gibco (Cat No.10576015), Foetal Bovine serum (FBS) from Sigma-Aldrich (F2442-500ML), and Colcemid solution from Gibco (Cat No. 15212012). For staining purposes, Giemsa powder from Merck was used. All other chemicals for preparing buffers and solutions used were of analytical grade.

\section{Study Design}

The study was a prospective observational study conducted in the affiliated institution for 2 years from March 2016 to February 2018. Both the male and female individuals with clinically suspected hematological cancer patients were included in our study without any age bar.

\section{Selection of Patients}

The present study was done on the people living in Kolkata, South 24 Pargana, North 24 Pargana, Howrah, and other adjoining areas in West Bengal, India. Samples were collected from 56 patients $(n=56)$ whose ages were in the range of 4 years to 63 years ( mean $=37.25 \pm 2, n=56$ ). The clinically defined types of the hematological cancers and the sex of the patients are mentioned in - Table $\mathbf{1}$.

\section{Collection of Sample}

Prior to collection of the sample, informed consent was obtained from all the patients after mentioning the purpose and impact of the study. In case of minors, the consent was obtained from their parents. Peripheral blood of $2 \mathrm{~mL}$ or bone marrow-aspirated cells were collected aseptically in prelabeled heparinized vials under the supervision of the trained persons. After collection, the vials were labeled appropriately, and all the necessary information related to medical purpose was documented. The vials were transferred to the cytogenetic laboratory as soon as possible with proper surveillance and care.

\section{Cytogenetic Analysis}

Cytogenetic analysis was performed based on phytohemaglutinin-stimulated peripheral blood lymphocyte cultures and without phytohemaglutinin-stimulated bone marrow cell aspiration culture of the respective patients. 
Table 2 Significant chromosomal instability observed in hematological cancer in present study

\begin{tabular}{|c|c|c|c|c|c|}
\hline SI. no. & Karyotype & Sample & $\begin{array}{l}\text { Clinical } \\
\text { type }\end{array}$ & Sex & Remark \\
\hline 1 & $46 X Y, t(9,22)(q 34, q 11)$ & $\mathrm{BM}$ and $\mathrm{PB}$ & CML & Male $=16$ & Classical Ph+ \\
\hline 2 & $46 X X, t(9,22)(q 34, q 11)$ & $\mathrm{BM}$ and $\mathrm{PB}$ & CML & Female $=9$ & Classical Ph+ \\
\hline 3 & 46XX,t(9;22)(q34:q11), i(12q) & PB & CML & Female & $\begin{array}{l}\mathrm{Ph}+\text { with associ- } \\
\text { ated chromosomal } \\
\text { abnormality }\end{array}$ \\
\hline 4 & 46XX,t(9;22;8)(q34:q11:p22) & PB & CML & Female & Variant $\mathrm{Ph}+$ \\
\hline 5 & $46 X Y, t(9 ; 22 ; 13)(q 34: q 11: q 14)$ & BM & CML & Male & Variant $\mathrm{Ph}+$ \\
\hline 6 & $46 X Y, t(9 ; 22)(q 34: q 11), \operatorname{inv}(7 p)(p 12 ; q 22)$ & PB & CML & Male & $\begin{array}{l}\mathrm{Ph}+\text { with associ- } \\
\text { ated chromosomal } \\
\text { abnormality }\end{array}$ \\
\hline 7 & 46XY,t(9;22;3)(q34:q11:p23) & PB & CML & Male & Variant $\mathrm{Ph}^{+}$ \\
\hline 8 & 46XY,t(9;22;1)(q34:q11:q22) & PB & CML & Male & Variant $\mathrm{Ph}+$ \\
\hline 9 & $47, \mathrm{XY}, \mathrm{t}(9 ; 22)(\mathrm{q} 34: \mathrm{q} 11),+8$ & PB & CML & Male & $\begin{array}{l}\mathrm{Ph}+\text { with associ- } \\
\text { ated chromosomal } \\
\text { abnormality }\end{array}$ \\
\hline 10 & $46, X Y, t(9 ; 22)(q 34: q 11), t(1: 6)(p 32: q 25)$ & BM & CML & Male & $\begin{array}{l}\mathrm{Ph}+\text { with associ- } \\
\text { ated chromosomal } \\
\text { abnormality }\end{array}$ \\
\hline 11 & 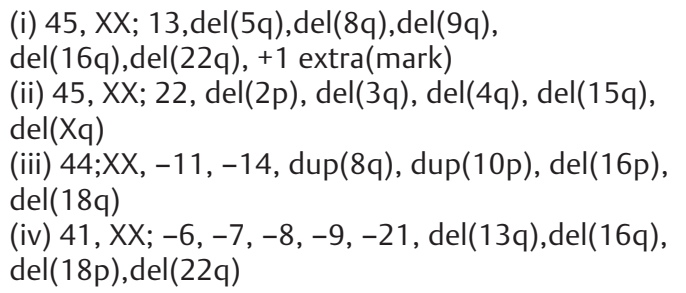 & PB & ALL & Female & Complex karyotypes \\
\hline 12 & 43, XX; -9, -20, -22, del(10q), del(14q), del(16p) & BM & ALL & Female & Hypodiploidy \\
\hline 13 & $44, X X ;+9,-12,-15,-16^{a}$ & PB & ALL & Female & Hypodiploidy \\
\hline 14 & $41, X Y ;-8,-9,-18,-21,-22, \operatorname{del}(2 p), \operatorname{del}(4 q), \operatorname{del}(11 q)$ & BM & ALL & Male & Hypodiploidy \\
\hline 15 & $45, \mathrm{XY} ;-8,-11,-18,-22$, mark +3 & PB & ALL & Male & Hypodiploidy \\
\hline 16 & $39, X Y ;-1,-9,-11,-12,-20,-22^{\mathrm{a}}$ & PB & ALL & Male & Hypodiploidy \\
\hline 17 & $\begin{array}{l}56, X X X, Y Y, 10,+4,+5,+6,+8,+11,+18,+21,+21 \\
\operatorname{der}(3,9)(q 10, q 10)\end{array}$ & BM & ALL & Male & Hyperdiploidy \\
\hline 18 & $46, \mathrm{XY} ;-5,-9,-10,-11,-12,+5$ marker chromosomes & PB & AML & Male & $\begin{array}{l}\text { Normal karyo- } \\
\text { types with marker } \\
\text { chromosomes }\end{array}$ \\
\hline
\end{tabular}

Abbreviations: '+'= Positive; ALL, acute lymphoblastic leukemia; AML, acute myelogenous leukemia; BM, bone marrow; CML, chronic myelogenous leukemia; PB, peripheral blood; Ph, Philadelphia chromosome.

${ }^{a}$ Complete 30 metaphase analysis was not possible in all cases due to poor morphology; however, total number of chromosomes and translocated chromosomes were countable and observable.

Lymphocyte culture, bone marrow cell aspiration culture and GTB-banding were performed following standard protocol as described by the AGT (Association of Genetic Technologists) Cytogenetic Laboratory Manual. ${ }^{8,9}$ Prepared slides were observed under light microscope (Olympus) with $10 \times 100$ magnification. Five fields were randomly observed for metaphase screening and 100 cells from each individual were observed and 25 metaphases were karyotyped. Karyotypes were described according to the International System for Cytogenetic Nomenclature (ISCN 2005).

\section{Statistical Analysis}

This study aimed to calculate the frequency of qualitative variables, such as, complex karyotypes, hypodiploidy, classical, and variant Philadelphia chromosome, and others, and their distribution with respect to hematological cancer in percentage. Other quantitative variables, such as, age. are expressed as mean standard.

\section{Ethics}

The study was approved by the Institute of Genetic Engineering ethical committee prior to the study (approval letter reference no: 03/GE/IGE/01/2016, 25 January 2016). All the procedures followed were in accordance with the ethical standards of the responsible committee on human experimentation and with the Helsinki Declaration of 1964, as revised in 2013. Informed consent was obtained from all the patients for being included in the present study. 
Table 3 Observed frequencies in percentage of qualitative variables in hematological cancer

\begin{tabular}{|l|l|l|l|}
\hline SI. no. & Qualitative variables in hematological cancer observed in current study & Observed no. & Percentage \\
\hline 1 & Rearranged chromosome & 41 & 73.21 \\
\hline 2 & Normal karyotypes & 15 & 26.78 \\
\hline 3 & Classical Philadelphia chromosome & 25 & 44.64 \\
\hline 4 & Philadelphia variant & 08 & 14.28 \\
\hline 5 & Hypodiploidy & 05 & 08.92 \\
\hline 6 & Hyperdiploidy & 01 & 01.78 \\
\hline 7 & Complex karyotype & 01 & 01.78 \\
\hline 8 & Karyotype with marker chromosomes & 01 & 01.78 \\
\hline 9 & CML & 42 & 75.00 \\
\hline 10 & AML & 07 & 12.50 \\
\hline 11 & ALL & 05 & 08.92 \\
\hline 12 & Clinically undiagnosed & 02 & 03.57 \\
\hline Total no of cases $(n=56)$ & & \\
\hline
\end{tabular}

Abbreviations: ALL, acute lymphoblastic leukemia; AML, acute myelogenous leukemia; CML, chronic myelogenous leukemia.

\section{Results}

In the present study, 56 suspected hematological cancer patients were studied. Among which 34 patients were male and 22 were female individuals. Cytogenetic evaluation revealed that out of 56 cases, 41 had rearranged chromosomes when 15 individuals showed normal karyotypes. Among 41 altered chromosome cases, 25 have classical Philadelphia chromosomes, 8 cases of Philadelphia variant chromosome, 5 cases of hypodiploidy, and single case each with hyperdiploidy and complex karyotypes. The karyotypes of each case, sex, sample for chromosome preparation, and clinically defined types of hematological cancer are depicted in - Table $\mathbf{2}$. The frequency of qualitative variables, that is, complex karyotypes, hypodiploidy, classical and variant Philadelphia chromosome, and their distribution with respect to hematological cancer in percentage are depicted in - Table 3.

\section{Discussion}

Leukemia or white blood cell disorder is one of the common hematological disorders after anemia, bleeding disorders such as hemophilia, and blood clotting disorder. ${ }^{10}$ Leukemia is mainly subdivided into two main groups like myelogenous leukemia or "myeloid" or "non-lymphocytic" leukemia and lymphocytic leukemia or "lymphoblastic," but both the types can be classified into chronic and acute subtypes. Acute leukemia is a clonal expansion of white blood cell precursors in the blood, bone marrow, and various extra medullary tissues. Acute lymphoblastic leukemia (ALL) comprises of neoplastic precursor cells committed to the B- or T-cell lineages. ${ }^{11} \mathrm{~B}$ lineage ALL is more frequent, accounting for $85 \%$ of childhood ALL and $75 \%$ of adult ALL. ${ }^{11}$ Acute myelogenous leukemia (AML), also known as acute nonlymphocytic leukemia, represents a group of clonal hematopoietic stem cell disorders in which cells fail to differentiate and over proliferate into the stem cell compartment, resulting in the accumulation of myeloblasts. ${ }^{12}$ In most of the cases, chronic lymphocytic leukemia often affects adults over the age of 55 years. It sometimes occurs in teenagers, but it almost never affects children. The 5-year survival rate is $75 \%$. In chronic lymphoblastic leukemia (CLL), B lymphocytes are affected. Generally B-cells originate in the bone marrow, develop in the lymph nodes, and normally fight infections by producing antibodies. ${ }^{13}$ Chronic myelogenous leukemia (CML) occurs mainly in adults. A very small number of children also develop this disease. It is a type of myeloproliferative disease associated with a characteristic chromosomal translocation called a Philadelphia translocation which is characterized by a cytogenetic aberration of reciprocal translocation between the long arm of chromosomes 9 and 22. About 85\% CML cases are Philadelphia positive. ${ }^{14}$ Chronic myelomonocytic leukemia (CMML) is another subtype with overlapping features of myeloproliferative neoplasm and myelodysplastic syndrome bearing clonal cytogenetic abnormalities in approximately $30 \%$ of patients. ${ }^{15,16}$

In the present study, among 56 suspected cases, 42 (75\%) individuals were clinically diagnosed as CML and among 42 cases, 33 (78.57\%) were found to have altered chromosomes, and finally among 33 cases, 25 (75.7\%) cases had classical Philadelphia positive variant and 8 (24.20\%) had Philadelphia variant. The Philadelphia variant cases involve translocation in chromosome numbers 1, 3, 6, 8, 11, 13 along with chromosome numbers 9 and 22 and the involved breakpoint regions are $1 \mathrm{p} 32,3 \mathrm{p} 23,6 \mathrm{q} 25,8 \mathrm{p} 22,11 \mathrm{q} 22$, and 13q14, respectively. Rather than translocation, occurrence of inversion and isochromosome formation observed on chromosome numbers 7 and 12 and trisomy 8 with Philadelphia-positive case was also observed in this study. According to various literature reviews, the chromosomal breakpoints of Philadelphia variants mainly occurs in Guanine-Cytosine (GC) rich part of the genome which is lightly banded during giemsa trypsin banding (GTB) technique. ${ }^{17}$ Generally, chromatin condensation and transcriptional activity are directly correlated with cytosine and guanine-rich portion of the genome. Mutation and 
natural cellular mechanism occur consequently within the genome, resulting chromosome breakage and finally leading to illegitimate recombination and translocation. ${ }^{17}$

Detailed molecular mechanism of variant Philadelphia chromosome and involvement of different chromosomes in varied breakpoint regions is still not clear, and it is often thought that for the evolution of variant Philadelphia chromosome, more than one molecular mechanism played a significant role. It could arise simultaneously in a three-way rearrangement or originate secondarily with the normal 9 to 22 translocation. The first mechanism could cause clonal variation and the second one may be similar to the classical Philadelphia chromosome but the clinical significance of both the classical and variant types was not even clear to the clinician. ${ }^{18,19}$ The variant Philadelphia chromosome may be simple or complex. If the deleted $22 \mathrm{q}$ region was translocated to other than chromosome 9, the type was a simple variant and when more than three chromosomes were involved, then the type was considered as complex variant. Recently with the advancement of molecular cytogenetic techniques, like microarray, several genes were identified for hematological cancer progression. Some of them directly participate in MAP (mitogen-activated protein) kinase pathway which was already known for CML pathogenesis. The BCR-ABL1 tyrosin kinase activities, the P13K/AKT signaling pathway in classical Philadelphia chromosome have established the causal relationship in between B-cell receptor/abel-like kinase translocation (BCR-ABL1) and CML. In addition to that, TP53 gene and two other genes, namely, MECOM and VRK1, thought to be responsible for blast crisis in $30 \%$ of CML cases. ${ }^{20,21}$

In the present study, among 56 suspected cases, 7 (12.5\%) individuals were clinically diagnosed as ALL, and all 07 $(100 \%)$ cases were found to have altered chromosomes and finally among 7 cases, 5 (71.47\%) cases show hypodiploidy, 1 (14.28\%) case of hyperdiploidy, and 1 (14.28\%) case had complex karyotypes. In the case of hypodiploidy, both structural and numerical chromosomal abnormalities like structural chromosomal deletion in both "p" and "q" arm of the chromosome, as well as whole chromosome deletion in variant chromosomes were found. In the hyperdiploidy case, the modal number increases due to presence of trisomy and tetrasomy along with whole chromosome deletion or monosomy and chromosome derivatives in variant autosomes and sex chromosome. ${ }^{22}$ The possible mechanism of hypo- and hyperdiploidy was an area of interest. Nondisjunction at the time of cell division was supposed to be the main possible cause of gain or loss of a whole chromosome, doubling of chromosomes from a near haploid karyotype and gain of chromosomes from a diploid karyotype during an abnormal cell cycle. ${ }^{23}$ Hyperploid karyotype generally arises by simultaneous gain of chromosomes from a diploid organism due to a single chromosomal abnormality. Different types of aneuploidy, like formation of micronucleus, lagging of chromosomal movement, or deletion of parts of chromosomes or telomeric loss, are the main chromosomal instabilities resulting genetic conformation produced by nondisjunction. ${ }^{24-28}$ According to different studies, chromosomal alteration or chromosomal instability had a great significance in the prognosis of hematological cancer. According to occurrence of chromosomal abnormality, patients with hematological cancers can be classified into three subgroups, that is, good, intermediate, and poor prognosis groups. Patients with the normal cytogenetic profile may even have submicroscopic genomic rearrangements which could not be detected by different molecular genetic analysis only. Among all identifiable chromosomal abnormality of hematological cancer, high hyperploids $(2 \mathrm{n}=51-65)$ had been associated with good prognosis. In these cases, the prognostic factors were clinically favorable and also independently favorable. ${ }^{29}$ Hyperdiploidy, that is, $2 \mathrm{n}=50$, had the most favorable prognostic value compared with other cytogenetically detected group. But the prognosis deteriorates as the chromosome number decreases. When chromosome number shows $(2 n=43-46)$, the prognosis was poor and as the number comes near to the haploidy $(2 n=30)$, then the prognosis also becomes worse and further management becomes difficult for the clinician. When hyperdiploidy or hypodiploidy is associated with structural chromosomal aberrations, the prognostic value was also poor. ${ }^{30-35}$ Among structural abnormalities, deletion and chromosomal translocation were known to be associated with poor prognosis. During the present study, the modal number of all hypodiploidy cases was between 40 and 46 chromosomes and 56 in the single case of hyperdiploidy. When there were more than one cell lines present within the same individuals, the situation was known as mosaicism and the representing karyotypes was known as complex karyotypes. Four different cell lines in different percentage within same individuals were observed in this current study. 36,37

Among 56 suspected hematological cases, 7 (12.5\%) individuals were clinically diagnosed as AML and among 7 cases, only 1 case (14.28\%) was found as altered chromosomes with marker chromosomes, monosomies within the same karyotypes, but total chromosome number remained 46. Other suspected hematological cancer cases were normal karyotypes without any microscopic chromosomal abnormalities. Detailed procedure of therapy or drug responsiveness after diagnosis and cytogenetic evaluation of chromosomal instability was not considered in the domain of present work. The patient follow-up study for all patients could not be considered within this study as few patients were referred to other hospitals or institutions, other oncologists, or transferred to private health care sectors by their relatives, and two cases ended in sad demises.

\section{Conclusion}

The present study showed the rare and varied chromosomal instabilities in clinically detected various hematological cancers cases in Kolkata and adjoining areas of West Bengal. The cytogenetic evaluation revealed classical and variant Philadelphia translocation including associated chromosomal abnormalities, complex karyotypes, mosaicism, hypodiploidy, and hyperdiploidy in a frequently manner in hematological malignancies. But, the adverse clinical, hematological, and cytogenetic parameters in patient with 
hematological cancers were more prevalent. If the analysis could be done at the time of remission and relapse, maintenance of proper patients' follow-up and finally detection of submicroscopic chromosomal rearrangements by advance molecular cytogenetic techniques in the selected cases were the most advisable strategy which help in a large number of patients to reach at a rational conclusion for prognostic stratification. In this way, the routine cytogenetic analysis could help the patients, as well as clinicians, for better understanding the neoplastic process and planning for appropriate management.

\section{Data Sharing Statement}

We cannot share any unpublished data with other laboratory or person.

\section{Funding}

This research received no specific grant from any funding agency in the public, commercial, or not-for-profit sectors. All the research work done by the affiliated institution funding.

\section{Conflict of Interest}

The authors declare that they have no conflict of interest to declare.

\section{Acknowledgments}

The first author acknowledges Institute of Genetic Engineering (IGE) for funding and affiliation. We are also thankful to other laboratory members and other associated persons of IGE for their enthusiastic participation.

\section{Reference}

1 Hanahan D, Weinberg RA. The hallmarks of cancer. Cell 2000;100(1):57-70

2 Diaz-Cano SJ. Tumor heterogeneity: mechanisms and bases for a reliable application of molecular marker design. Int J Mol Sci 2012;13(2):1951-2011

3 Bhatt AN, Mathur R, Farooque A, Verma A, Dwarakanath BS. Cancer biomarkers - current perspectives. Indian J Med Res 2010;132:129-149

4 Garnis C, Buys TPH, Lam WL. Genetic alteration and gene expression modulation during cancer progression. Mol Cancer 2004;3:9

5 Vayen B, Thomay K, Schlbelberger B. Induction of chromosomal instability via Telomer dysfunction and epigenetic alteration in myeloid neoplasia. Cancer 2013;5(3):857-874

6 Rowe JM. Prognostic factors in adult acute lymphoblastic leukaemia. Br J Haematol 2010;150(4):389-405

7 Silva ML, Ornellas de Souza MH, Ribeiro RC, et al. Cytogenetic analysis of 100 consecutive newly diagnosed cases of acute lymphoblastic leukemia in Rio de Janeiro. Cancer Genet Cytogenet 2002;137(2):85-90

8 Moorhead PS, Nowell PC, Mellman WJ, Battips DM, Hungerford DA. Chromosome preparations of leukocytes cultured from human peripheral blood. Exp Cell Res 1960;20:613-616

9 Seabright M. A rapid banding technique for human chromosomes. Lancet 1971;2(7731):971-972

10 World Health Organization World Cancer Report 2014. Switzerland: World Health Organization; 2014

11 Graux C. Biology of acute lymphoblastic leukemia (ALL): clinical and therapeutic relevance. Transfus Apheresis Sci 2011;44(2):183-189
12 Alcalay M, Orleth A, Sebastiani C, et al. Common themes in the pathogenesis of acute myeloid leukemia. Oncogene 2001;20(40):5680-5694

13 Cheson BD, Bennett JM, Grever M, et al. National Cancer Institute-sponsored Working Group guidelines for chronic lymphocytic leukemia: revised guidelines for diagnosis and treatment. Blood 1996;87(12):4990-4997

14 Pasternak G, Hochhaus A, Schultheis B, Hehlmann R. Chronic myelogenous leukemia: molecular and cellular aspects. J Cancer Res Clin Oncol 1998;124(12):643-660

15 Patnaik MM, Tefferi A. Chronic myelomonocytic leukemia: 2018 update on diagnosis, risk stratification and management. Am J Hematol 2018;93(6):824-840

16 McCullough KB, Patnaik MM. Chronic myelomonocytic leukemia: a genetic and clinical update. Curr Hematol Malig Rep 2015;10(3):292-302

17 Fisher AM, Strike P, Scott C, Moorman AV. Breakpoints of variant 9;22 translocations in chronic myeloid leukemia locate preferentially in the CG-richest regions of the genome. Genes Chromosomes Cancer 2005;43(4):383-389

18 Chauffaille ML. Cytogenetics and FISH monitoring CMLduring tyrosine kinase inhibitors treatment. Rev Bras Hematol Hemoter 2008;30(1):13-19

19 Yehuda O, Abeliovich D, Ben-Neriah S, et al. Clinical implications of fluorescence in situ hybridization analysis in 13 chronic myeloid leukemia cases: Ph-negative and variant Ph-positive. Cancer Genet Cytogenet 1999;114(2):100-107

20 Kelman Z, Prokocimer M, Peller S, et al. Rearrangements in the p53 gene in Philadelphia chromosome positive chronic myelogenous leukemia. Blood 1989;74(7):2318-2324

21 Huret JL, Ahmad M, Arsaban M, et al. Atlas of genetics and cytogenetics in oncology and haematology in 2013. Nucleic Acids Res 2013;41(Database issue):D920-D924

22 Amare P, Gladstone B, Varghese C, Pai S, Advani S. Clinical significance of cytogenetic findings at diagnosis and in remission in childhood and adult acute lymphoblastic leukemia: experience from India. Cancer Genet Cytogenet 1999;110(1):44-53

23 Panzer-Grümayer ER, Fasching K, Panzer S, et al. Nondisjunction of chromosomes leading to hyperdiploid childhood B-cell precursor acute lymphoblastic leukemia is an early event during leukemogenesis. Blood 2002;100(1):347-349

24 Bovery T. Concerning the origin of malignant tumor by TheoderBoveri. J Cell Sci 2008;121:1-84

25 Negrini S, Gorgoulis VG, Halazonetis TD. Genomic instability-an evolving hallmark of cancer. Nat Rev Mol Cell Biol 2010;11(3):220-228

26 Jallepalli PV, Lengauer C. Chromosome segregation and cancer: cutting through the mystery. Nat Rev Cancer 2001;1(2):109-117

27 Santaguida S, Amon A. Short- and long-term effects of chromosome mis-segregation and aneuploidy. Nat Rev Mol Cell Biol 2015;16(8):473-485

28 Holland AJ, Cleveland DW. Losing balance: the origin and impact of aneuploidy in cancer. EMBO Rep 2012;13(6):501-514

29 Ito C, Kumagai M, Manabe A, et al. Hyperdiploid acute lymphoblastic leukemia with 51 to 65 chromosomes: a distinct biological entity with a marked propensity to undergo apoptosis. Blood 1999;93(1):315-320

30 Hiroyuki T, Ichiro M, Yasuhiko K. Why does childhood acut lymphoblastic leukemia with hyperdiploidy show a fevour prognosis? Cancer Genet 1990;50(2):273-275

31 Bloomfield CD, Goldman AI, Alimena G, et al. Chromosomal abnormalities identify high-risk and low-risk patients with acute lymphoblastic leukemia. Blood 1986;67(2):415-420

32 Pui CH, Williams DL, Roberson PK, et al. Correlation of karyotype and immunophenotype in childhood acute lymphoblastic leukemia. J Clin Oncol 1988;6(1):56-61 
33 Fenaux P, Lai JL, Morel P, et al. Cytogenetics and their prognostic value in childhood and adult acute lymphoblastic leukemia (ALL) excluding L3. Hematol Oncol 1989;7(4):307-317

34 Look AT, Roberson PK, Williams DL, et al. Prognostic importance of blast cell DNA content in childhood acute lymphoblastic leukemia. Blood 1985;65(5):1079-1086

35 Smets LA, Homan-Blok J, Hart A, et al. Prognostic implication of hyperdiploidy as based on DNA flow cytometric measurement in childhood acute lymphocytic leukemia-a multicenter study. Leukemia 1987;1(3):163-166

36 Zemanova Z, Michalova K, Sindelarova L, et al. Prognostic value of structural chromosomal rearrangements and small cell clones with high hyperdiploidy in children with acute lymphoblastic leukemia. Leuk Res 2005;29(3):273-281

37 Debatin KM, Stahnke K, Fulda S. Apoptosis in hematological disorders. Semin Cancer Biol 2003;13(2):149-158 